\title{
Cultivation of microalgae in wastewater: A review
}

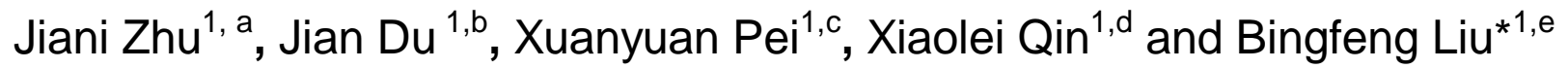 \\ ${ }^{1}$ State Key Laboratory of Urban Water Resource and Environment, Harbin Institute of Technology, \\ No.73 Huanghe Road, 2nd Campus of HIT Box 2614, Harbin 150090, Heilongjiang, China \\ a1112710131@hit.edu.cn, bpxyzby@126.com, ${ }^{\mathrm{c}} 274909984 @ q q . c o m$, \\ d1112710132@hit.edu.cn, ${ }^{\mathrm{e}} \mathrm{lbf} @$ hit.edu.cn
}

${ }^{*}$ Corresponding author. Tel/Fax:+86 451 86282008. E-mail: Ibf@hit.edu.cn (B.-F. Liu)

Keywords: microalgae; cultivation; wastewater; biodiesel

Abstract. Microalgae have great potential as the feedstock of renewable energy. Nevertheless, the high cost of microalgae cultivation hinders its development economically, so inexpensive cultural mediums are supposed to be found for the large-scale application of microalgae. Wastewater contains large amount of organic and inorganic compounds, which provides nutrition for the cell growth and lipid accumulation of microalgae. Here we will review the systems of microalgae cultivation and utilize wastewater as a feedstock for biodiesel production. It illustrates that the integration of microalgae cultivation and wastewater treatment could improve water quality and accumulate lipid simultaneously.

\section{Introduction}

Energy and water are the most focused topics in the $21^{\text {th }}$ century. On one hand, fossil fuel is non-renewable and will be exhausted in nearly 70 years [1], which makes the energy problems more imminent. Meanwhile, fossil fuel caused tremendous pressure on the environment. Its application increases air pollution, haze, acid rain [2] and greenhouse effect, which raises the temperature of the surface of our earth and causes the melt of polar ice as well as sea-level rise. Therefore, changing the energy structure and looking for clean, renewable new energy is necessary and it is also the faced challenges of global energy issues. Microalgae, as feedstock of the third generation of biodiesel, can survive in the harsh surroundings and degrade organic matter, remove nitrogen, phosphorus and toxic metals efficiently in sewage [3]. On the other hand, traditional wastewater treatment such as activated sludge process has some drawbacks, which has difficulties in the removal of nitrogen and phosphorus. In addition, the produced excess activated sludge is usually disposed as waste [4].

Recently, the microalgae have been applied widely for wastewater treatment. It shows great potential because of the excellent performance of wastewater treatment and the lower cost compared with traditional process. Using microalgae for treating wastewater not only purifies water, but also achieves the energy recovery. Furthermore, the microalgae can be used as fertilizer or as animal feed, which is avoid of generating additional pollutants like sludge byproducts [5]. Alternatively, microalgae biomass can be utilized as bioplastic, pigments, nutraceuticals and so on.

\section{Microalgae cultivation and cultural systems}

Microalgae are unicellular or filamentous algae with diameters less than $2 \mathrm{~mm}$ [6]. As one of the oldest living creatures, they can be easily found in freshwater and marine. Oleaginous microalgae can convert carbon sources like carbon dioxide, carbohydrates and common fats into a large quantity of fat stored inside (>20\% dry weight) under certain conditions. These microscopic photosynthetic organisms are classified into three groups: 1) Autotrophic: They only need $\mathrm{CO}_{2}, \mathrm{~N}, \mathrm{P}$ and light to grow normally and accomplish nutrient accumulation. This subcategory includes photoautotrophs and chemoautotrophs, which require solar light and external organic compounds respectively as source of energy. 2) Heterotrophic: They get energy by means of oxidizing organic compounds, including 
photo-heterotrophs and chemo-heterotrophs. 3) Mixotrophic: Using different sources of carbon and energy for their growth [7].

Microalgae have the irreplaceable advantages of high lipid content, short growth cycle and easy cultivation and so on. By transesterification, microalgae oil can be transferred to biodiesel, which is environmental and safe. So far, the number of microalgae of which the fatty acid has been measured has been up to hundreds. Although there are oils and fats in algae cells generally, the content differs significantly, even with the same species. Table 1 shows the constituents of several algae.

Table 1. Biochemical constituents of several algae

\begin{tabular}{cccc}
\hline Species & Protein (\% dry weight) & Carbohydrate $(\%$ dry weight) & Lipid (\% dry weight) \\
\hline Scenedesmus dimorphus & $8-18$ & $21-52$ & $16-40$ \\
Prymnesium parvum & $28-45$ & $25-33$ & $22-39$ \\
Chlorella vulgaris & $51-58$ & $12-17$ & $14-22$ \\
Spirogyra sp. & $6-20$ & $33-64$ & $11-21$ \\
Chlamydomonas rheinhardii & 48 & 17 & 21 \\
Scenedesmus obliquus & $50-56$ & $10-17$ & $12-14$ \\
Porphyridium cruentum & $28-39$ & $40-57$ & $9-14$ \\
Dunaliella bioculata & 49 & 4 & 8 \\
Dunaliella salina & 57 & 32 & 6 \\
\hline
\end{tabular}

Cultivation mode. Rich nutrients and salts facilitate the growth of microalgae. Several physicochemical and biological factors such as light, temperature, $\mathrm{pH}$ and nutrition have impacts on algal growth, among which the availability of light and carbon are the most significant factors. The process of photosynthesis provides a lot of inorganic carbon, which produces glucose by microalgae when combining with solar light [8]. A study has shown that the average concentration of biomass may reach $180 \%$ with the photosynthetic period increasing from 6 to $12 \mathrm{~h}$ [9]. Microalgae can be grouped into four categories due to their metabolism.

Photoautotrophic mode. As the most widely used cultivation method for microalgae, photoautotrophic metabolism occurs during the photosynthetic period that microalgae synthesize chemical energy using light as the source of energy and inorganic carbon as the source of carbon. The major advantage of using autotrophic cultivation is that the carbon dioxide can be consumed as carbon source for the cell growth and oil production. Therefore, the scale-up cultivation systems of microalgae, such as open ponds and raceway ponds, are usually operated under the conditions of phototrophic cultivation.

Heterotrophic mode. The phenomenon of microalgae growth by using organic carbon as source of energy and carbon is named heterotrophic cultivation. This type of cultivation gets rid of the light limitation and it hinders high cell density during phototrophic period in large-scale photobioreactors. It often combines various industrial processes together, such as the combination of wastewater treatment and biofuel production [10]. Studies have also shown that heterotrophic cultivation is a more effective method since it is easier to scale up.

Mixotrophic mode. The cultivation of microalgae using both organic compounds and inorganic carbon as source of carbon for its growth called mixotrophic cultivation, which means that microalgae can be cultured under phototrophic or heterotrophic circumstance, or both. Microalgae of mixotrophic cultivation are rarely used in oil production [11], but this kind of cultivation mode has many advantages because the lack of dark respiration.

Photoheterotrophic mode. Photoheterotrophic cultivation can be regard as the combination of heterotrophy and phototrophy, which means that the microalgae need light as energy source while use organic compounds as carbon source. The source of photoheterotrophic cultivation is light while mixotrophic cultivation could use organic compounds to serve the same purpose.

Cultural systems. Microalgae can be cultivated in different types of systems, of which the main systems contain open-air ponds and closed photobioreactors (PBRs). Open reactors required of direct sunlight are usually built outdoors normally while closed cultures can be either indoors or outdoors [12]. Several factors such as the cost of land, the source of carbon and the species of algae should be taken into consideration when it was selected of an appropriate cultivation system. 
Open-air ponds. Raceway ponds are shallow ponds, of which the circuit of parallel channels consisting microalgae, water and nutrients circulate. In order to be better exposed to sunlight, raceway ponds are very shallow to improve the sunlight spread. Compared with other systems, open systems have many advantages such as simplicity. Besides, the costs of construction and production are lower. Moreover, the open systems can deal with large amounts of carbon dioxide and they don't need cooling or supersaturated oxygen, which avoid the production of biofuels in the closed system affected by the oversaturation oxygen. However, the length of the ponds ranges from 10 to $100 \mathrm{~m}$, the width of ponds varies from 1 to $10 \mathrm{~m}$ and the depth of ponds is 0.20 to $0.50 \mathrm{~m}$. In general, the area of ponds can be about to 0.5 hectares or larger [13]. Apparently, the construction and operation of open ponds are expensive unless the land and water are merely free. In addition, exposed to the air, microalgae might be polluted by external species with the temperature and the concentration of water affected by weather fluctuation. Furthermore, there is low cell density in these devices, which contribute to the high price of harvesting process. There are applications of the combination of open runway with wastewater treatment, such as high rate algal ponds (HRAP). The process of microalgae growth can consume nutrients in the sewage, which is economically feasible and environmentally friendly. By this method, the algae produce biodiesel while the wastewater is being treated.

Closed photobioreactors. Photobioreactors usually refer to transparent tubes made of glass or plastic, to ensure the light exposed homogeneously through the entire culture. According to the shapes, closed photobioreactors can be classified into tubular, flat plate and vertical or horizontal column [14]. They have advantages in terms of high efficiency and biomass productivity, short harvest times and high surface-to-volume ratios. Meanwhile, compared with open systems, closed photobioreactors reduce contamination risks and can be used to cultivate a greater range of algal species. Additionally, they can use sewage wastewater or flue gas, which is beneficial for $\mathrm{CO}_{2}$ reduction. However, they are much more expensive to construct. There are several limitations in photobioreactors, for example, the capital of output and the costs of operation. The complex systems need to avoid the accumulation of oxygen and the damage caused by shear stress. Several studies have compared the difference in types of photobioreactors and suggested improvements for classic designs, such as hybrid systems that combine photobioreactors with open ponds, and floating photobioreactors that can operate beneath water rather than land [15]. The cultivation of different microalgae is shown in table 2.

Table 2. Microalgae cultivation in closed photobioreactors

\begin{tabular}{cccc}
\hline Algae taxon & Type of photobioreactor & Biomass concentration & Reference \\
\hline Aphanothece microscopica & Bubble column & $0.77 \mathrm{~g} / \mathrm{L} / \mathrm{d}$ & {$[16]$} \\
Botryococcus braunii & Biofilm & $0.71 \mathrm{~g} / \mathrm{m} 2 / \mathrm{d}$ & {$[17]$} \\
Chlorella sp. & Airlift & $0.21 \mathrm{~g} / \mathrm{L} / \mathrm{d}$ & {$[18]$} \\
Chlorella vulgaris & Airlift & $0.28-0.89 \mathrm{~g} / \mathrm{L} / \mathrm{d}$ & {$[19]$} \\
Dunaliella tertiolecta & Flat plate & $3.42 \mathrm{~g} / \mathrm{d}$ & {$[20]$} \\
Phaeodactylum cruentum & Tubular & $35 \mathrm{~g} / \mathrm{m} 3 / \mathrm{d}$ & {$[21]$} \\
\hline
\end{tabular}

\section{Applications of microalgae in wastewater.}

With society and industry developed, the discharge of sewage increases gradually, of which the nitrogen, phosphorus and inorganic salts can be the inexpensive nutrition for the growth of microalgae. Over the past few decades, tremendous efforts have been made to microalgae cultivation in wastewaters. Studies have shown that microalgae can remove nitrogen, phosphorus of high concentration while they utilize nutrient synthesize useful biomass by photosynthetic conversion of solar energy. Moreover, they can remove toxins and heavy metals from wastewater. However, the major bottleneck of microalgae in large-scale cultivation is the expensive cost, of which the raw material occupies $70 \%$ [22]. Therefore, it is necessary to combine algae culture technology with wastewater treatment, which not only achieves the efficient and harmless purification of wastewater, but also reduces the cost of microalgae culture medium and recycles high-value algae biomass. 
Municipal wastewater. Wastewater has potential to supply the nutrition for survival of various organisms as medium. Among the sources of wastewater such as municipal, industrial and agricultural wastewater etc., the former one has more potential for microalgae growth because it contains more compounds. It is highly possible to use wastewater as medium to culture microalgae due to enormous quantity of daily wastewater.

Previous studies were focused on the cultivation of microalgae in wastewater collected from different units of the municipal wastewater treatment plant. Researches showed that Chlorella and Scenedesmus of various species provide very high efficiency in wastewater treatment, which almost removed ammonia, nitrate and total phosphorus completely from secondary treated wastewater in many cases. A Chlorella species identified in India [23] was able to grow well in high concentrations of raw sewage and dominated the subsequent stages in the oxidation pond system. Analysis has found that this species can utilize a variety of organic carbon substrates, adapt to a wide $\mathrm{pH}$ range and the presence of salt. It grew heterotrophically in the dark while mixotrophically in the light. Moreover, it can use either nitrate or ammonia. To sum up, the wastewater treatment efficiency increases when employing microalgae-bacterial system [24] and microalgae show the great potential for high biomass productivity in wastewater treatment system.

Industrial wastewater. Compared with others, there are more heavy metal pollutants in the majority of industrial wastewater while less nitrogen or phosphorus. The heavy metal can be removed by biosorption, accumulation, excretion and purification of microalgae because the cell wall of microalgae is negatively charge and it has high viscosity and large surface area while the cell membrane of microalgae has high selectivity and semipermeability. For higher removal efficiency, it is crucial to select the microalgae strains of high metal sorption capacity. Nevertheless, a few studies have been done upon the mental sorption of algal species. A recent study has manifested that the potential for the generation of algal biomass in wastewater from carpet mill effluent. Though there were chemicals, pigments, inorganic elements including low concentrations of metals in the wastewater with relatively low concentrations of nitrogen and phosphorus, the freshwater microalgae B. braunii and Chlorella saccharophila and a marine alga Pleurochrysis carterae, grew particularly well in the untreated wastewater [25].

Agricultural wastewater. Over the past decade, livestock have transformed from small to large scale, which made up the majority of agricultural wastewater. Besides, agricultural wastewater also contains herbicides, fungicides and insecticides, which may increase the difficulty in wastewater treatment. Compared to municipal wastewater, agricultural wastewater has high concentration of nitrogen and phosphorus. However, studies have demonstrated microalgae has the ability to grow in agricultural wastewater efficiently and remove nitrogen and phophorus from manure-based wastewater [26]

Wastewater as a feedstock for biodiesel production. Several studies have been conducted on simultaneous wastewater treatment and biodiesel production. Ren et al. [27] selected a novel green microalga Scenedesmus sp. strain R-16, which can grow well heterotrophically in artificial wastewater without light and reached $43.4 \%$ and $3.46 \mathrm{~g} \mathrm{~L}^{-1}$ for total lipid content and biomass respectively. Xin et al. [28] tested the ability of a freshwater microalga, Scenedesmus sp. LX1 to remove nutrients and accumulate lipid while growing in secondary effluent. The results showed that Scenedesmus sp. LX1 adapted better to secondary effluent and achieved the highest biomass $\left(0.11 \mathrm{~g} \mathrm{~L}^{-1}\right.$, dry weight $)$ and lipid content (31-33\%, dry weight) and inorganic nutrients could be efficiently removed by over $98 \%$ in 10 days. Hu et al. [29] examined the effect of exogenous $\mathrm{CO}_{2}$ on the growth and lipid accumulation of an Auxenochlorella protothecoides (UMN280) as well as nutrient removal from concentrated municipal wastewater stream. The lipid content can reach $20.58 \%$ with $5 \% \mathrm{CO}_{2}$ and the efficiencies of nutrient removal on $\mathrm{COD}, \mathrm{NH}_{3}-\mathrm{N}, \mathrm{TN}$, and $\mathrm{PO}_{4}-\mathrm{P}$ was high.

There were studies using industrial wastewater to assimilate nitrogen and phosphorus with lipid production [30]. Senthil Chinnasamy et al. [25] separated a strain of algae from the carpenter wastewater treatment plant. Evaluating the algae in a runway pool of which the volume was $950 \mathrm{~L}$. It was found that the algae had potential to produce 9.2-17.8 tons biomass per hectare annually and the lipid content was $6.82 \%$. Hongyang et al. [31] cultivated Chlorella pyrenoidosa using soybean process 
wastewater without any additional nutrients in fed-batch culture. After $120 \mathrm{~h}$, the alga was able to remove $77.8 \%, 88.8 \%$ and $70.3 \%$ of soluble chemical oxygen demand, total nitrogen and total phosphate, respectively. Meanwhile, $C$. pyrenoidosa attained an average biomass productivity of 0.64 $\mathrm{g} \mathrm{L}^{-1} \mathrm{~d}^{-1}$ and lipid content of $37.0 \%$.

M.K.Kim et al. [32] used a medium with broiler fermentation wastewater added to culture Scenedesmus in shake flasks, the growth rates and the dry weight can be 3 times and 2.6 times of those in control groups, respectively. A study of Johnson, M.B. et al. [33] showed that Chlorella sp. in dairy manure wastewater had very high biomass as well as fatty acid yield. Orpez, R. et al. [34] found that Botryococcus braunii cultivated in sewage (secondarily treated) as tertiary treatment unit can acquire good performance with $17 \%$ lipid accumulation. There were also other researches suggested that the industrial wastewater can be subjected to dilution or mixing with organic compounds will promote the growth of microalgae [35,36].

\section{Conclusions}

Microalgae, as the most promising feedstock for biodiesel, have irreplaceable advantages of rapid growth and high lipid content. However, the cost of cultivation is expensive, which limits its industrial production. Sewage, industrial and agricultural wastewater can supply nutrition as medium for the growth of microalgae. Therefore, the combination of algae culture and wastewater treatment not only achieves the efficient removal of pollution and energy recovery, but also reduces the cost of microalgae culture medium, which provides a strategy for simultaneous wastewater treatment and energy recovery.

\section{Acknowledgements}

This work was financially supported by the National Natural Science Fund of China (No. 51478139).

\section{References}

[1] E. Stephens, I.L. Ross, J.H. Mussgnug, L.D. Wagner, M.A. Borowitzka, Posten, O. Kruse, B. Hankamer: Trends Plant Sci. Vol. 15 (2010), p. 554-564.

[2] N. Pragya, K.K. Pandey, P.K. Sahoo: Renew. Sust. Energ. Rev. Vol. 24 (2013), p. 159-171.

[3] Ahluwalia, S.S., D.Goyal: Bioresour. Technol. Vol. 98 (2007), p. 2243-2257.

[4] Silva, T.F.C.V., Silva, M.E.F., Cunha-Queda, A.C., Fonseca, A., Saraiva, I., Boaventura, R. A.R., Vilar, V.J.P.: Chem. Eng. J. Vol. 28 (2013), p. 850-866.

[5] Munoz, R., Guieysse, B.: Water Res. 40 (2006), p. 2799-2815.

[6] Ramón Piloto-Rodríguez, Yisel Sánchez-Borrotoa, Eliezer Ahmed Melo-Espinosaa, Sebastian Verhelstb: Renew Sust Energ Rev. Vol. 69 (2017), p. 833-842.

[7] L. Brennan, P. Owende: Renew. Sust. Energ. Rev. Vol. 14 (2010), p. 557-577.

[8] K. Larsdotter: Waste Manag. Res. Vol. 62 (2006), p. 31-38.

[9] S.Y. Ip, J.S. Bridger, C.T. Chin, W.R.B. Martin: Water Res. Vol. 16 (1982), p. 621-632.

[10] O. Perez-Garcia, F.M.E. Escalante, L.E. de-Bashan: Water Res. Vol. 45 (2011), p. 11-36.

[11] C.Y. Chen, K.L. Yeh, R. Aisyah, D.J. Lee: Bioresour. Technol. Vol. 102 (2011), p. 71-81.

[12] A. Bahadar, M.B. Khan: Renew. Sust. Energ. Rev. Vol. 27 (2013), p. 128-148.

[13] K. Sompech, Y. Chisti, T. Srinophakun: Biofuels. Vol. 3 (2012), p. 387-397.

[14] L. Brennan, P: Renew. Sust. Energ. Rev. Vol. 14 (2010), p. 557-577. 
[15] Antoine Soulièsa, Jérémy Pruvostb, Cathy Castelaina, Teodor Burghelea: J NON-NEWTON FLUID. Vol. 231(2016), p. 91-101.

[16] Jacob-Lopes E, Scoparo CHG, LMCF Lacerda, Franco TT: CHEM ENG PROCESS. Vol. 48 (2009), p. 306-310.

[17] Ozkan A., Kinney K., Katz L., Berberoglu H: Bioresource Technol. Vol. 114(2012), p. 542-548.

[18] Zhang K, Kurano N, Miyachi S: Bioprocess and Biosystems Engineering Vol. 25 (2002), p. 97-101.

[19] Feng Y, Li C, Zhang D: Bioresource Technol.Vol. 102 (2011), p. 101-105.

[20] Zemke P.E. Mathematical modeling of light utilization and the effects of temperature cycles on productivity in a steady-state algal photobioreactor; (2010).

[21] Masojídek J, Papác ek Š, Sergejevova M, Jirka V, C ervený J, Kunc J: J APPL PHYCOL. Vol. 15 (2003), p. 239-248.

[22] Venkata Mohan, S., Prathima Devi, M., Mohanakrishna, G., Amarnath, N., Lenin Babu, M., Sarma, P.N: Bioresour. Technol. Vol. 102 (2011), p. 1109-1117.

[23] Bhatnagar A, Bhatnagar M, Chinnasamy S, Das KC: Appl Biochem Biotechnol. Vol. 161 (2010), p. 523-536.

[24] Su Y, Mennerich A: Water Res. Vol. 45 (2011), p. 3351-3358.

[25] Chinnasamy, S., Bhatnagar, A., Hunt, R.W., Das, K.C: Bioresour. Technol. Vol. 101 (2010), p. 3097-3105.

[26] Gonzalez, L.E., Canizares, R.O., Baena, S: Bioresour. Technol. Vol. 60 (1997), p. 259-262.

[27] Ren, H.Y., Liu, B.F., Ma, C., Zhao, L., Ren, N.Q.: Biotechnol. Biofuels. Vol. 6 (2013), p. 143.

[28] Xin, L., Hong ying, H., Jia, Y.: N. Biotechnol. Vol. 27 (2010), p. 59-63.

[29] Hu, B., Min, M., Zhou, W., Li, Y., Mohr, M., Cheng, Y: Appl. Microbiol. Biotechnol. Vol. 166 (2012), p. 1661-1673.

[30] Li, F.W., Pei, C., Ai, P.H., Chi, M.L: Bioresour. Technol. Vol. 113 (2012), p. 14-18.

[31] Hongyang, S., Yalei, Z., Chunmin, Z., Xuefei, Z., Jinpeng, L: Bioresour. Technol. Vol. 102 (2011), p. 9884-9890.

[32] Kim M K, Park J W, Park C S: Bioresour Technol. Vol. 98 (2008), p. 2220-2228.

[33] Johnson, M.B., Wen, Z.Y: Appl. Microbiol. Biotechnol. Vol. 85 (2010), p. 525-534.

[34] Orpez. R., Martinez. M.E., Hodaifa. G., El Yousfi. F., Jbari. N., Sanchez. S.: Desalination. Vol. 246 (2009), p. 625-630.

[35] Li C, Yang H, Xia X, Li Y, Chen L, Zhang M: Bioresour Technol. Vol. 127 (2013), p. 248-55.

[36] Kothari R, Pathak VV, Kumar V, Singh DP: Bioresour Technol. Vol. 116 (2012), p. 466-470. 